protection against infection of Covid 19 in comparing to other receiving the other biologicals or conventional Therapy or not.

Objectives: Our objective was to determine if tocolizumab had a primary protection against SARS-COV2 in comparing to patients were on other biologics. Methods: A Retrospective study was done among 100 patients with rheumatic diseases (Group 1: 10 on tocilizumab, Group2: 40 (30 on antiTNF and 10 on IL17 antagonist), Group3: on cDMARDs) from March to Dec 2020. Detailed history, clinical presentation, laboratory, computed tomography of chest and PCR for covid 19 if presented among infected patients.

Results: Among 100 patients of median age 21, 19 patient (19\%) had infected with Covid 19 (2 on tocilizumab, 6 on antiTNF, 1 on IL17 antagonist, 10 on cDMARDs). Symptoms were reported fever $(89.4 \%)$, Fatigue $(78.9 \%)$, myalgia $(78.9 \%)$, Headache $(73.7 \%)$, Dyspnea $(52.6 \%)$, Cough $(47.3 \%)$, chest pain (26.3\%) and hypoxia (5\%). CT findings were positive in 10 patients $(52.63 \%)$ while PCR was done and positive in 15 out of 19 (78.94\%). Nevertheless, there was no significant statistically difference between groups according to infection with SARS-COV2. $(\mathrm{p}=0.704)$

Conclusion: Tocilizumab is not shown to be protective against SARS-COV2 (COVID 19) infection compared to other biological or cDMARDs in patients with rheumatic diseases.

REFERENCES:

[1] Tleyjeh IM, Kashour Z, Damlaj M, et al. Efficacy and safety of tocilizumab in COVID-19 patients: A living systematic review and meta-analysis. Clinical Microbiology and Infection. 2020 Nov 5.

[2] Tsai, A., Diawara, O., Nahass, R.G. et al. Impact of tocilizumab administration on mortality in severe COVID-19. 2020;Sci Rep 10:19131

Disclosure of Interests: None declared.

DOI: 10.1136/annrheumdis-2021-eular.461

\section{AB0655 RHEUMATOID ARTHRITIS ACTIVITY BEFORE AND AFTER COVID-19 LOCKDOWN PERIOD}

M. Jordhani', D. Ruci2, F. Skana2, E. Memlika2. ${ }^{1}$ UHC Mother Teresa, Tirana, Albania, Internal Medicine, Tirana, Albania; ${ }^{1}$ UHC Mother Teresa, Tirana, Albania, Internal Medicine, Tirana, Albania

Background: The COVID-19 global pandemic has had a great impact on world population due to morbidity, mortality and restriction measures in order to stop the progression of COVID-19.

Patients with rheumatic and musculoskeletic diseases, and especially rheumatoid arthritis (RA) patients, being one of the vulnerable classes of chronic patients, were recommended to follow the government's rules ${ }^{1}$.

Objectives: The aim of this study was to evaluate DAS-28-ESR in patients with rheumatoid arthritis before and after lockdown period.

Methods: This is a multi-center observational study including 85 patients which were evaluated before and after lockdown for their disease activity score according to DAS-28-ESR score. They had been diagnosed with rheumatoid arthritis more than 5 years ago. A thorough physical examination was performed before and after the lockdown period. It included examination of tender and swollen joints and patient's global health. They were completed with all required laboratory data, including erythrosedimentation rate. For a more accurate calculation, DAS-28-ESR was used in an electronic version. Patients with other inflammatory or infective diseases were excluded from the study. All data were statistically evaluated using statistical tests such as t-student test.

Results: The first group (the one before lockdown) had an average DAS-28-ESR of 4.7 while after the lockdown period, the average DAS-28-ESR was 5.16.

After statistically evaluating all data, it was found that there exists a significant difference between DAS-28-ESR score before and after COVID-19 lockdown $(p=0.0011)$

Conclusion: Our study showed that lockdown period due to COVID-19 pandemic, has aggravated disease activity in patients with Rheumatoid Arthritis. This may be consequence of various causes such as physical inactivity and difficulty to follow-up or to take the medication properly.

\section{REFERENCES:}

[1] Landewé RB, Machado PM, Kroon F, et al, EULAR provisional recommendations for the management of rheumatic and musculoskeletal diseases in the context of SARS-CoV-2, Annals of the Rheumatic Diseases 2020;79:851-858.

Disclosure of Interests: None declared.

DOI: 10.1136/annrheumdis-2021-eular.576

\section{AB0656 SARS-COV-2 INFECTION IN PATIENTS WITH RHEUMATIC DISEASE: A TERTIARY SINGLE-CENTER EXPERIENCE}

A. Halbac ${ }^{1}$, B. Balan ${ }^{1}$, C. Tamas ${ }^{1}$, M. M. Tamas ${ }^{1,2}$, I. Felea ${ }^{1}$, I. Filipescu ${ }^{1,2}$ L. Damian ${ }^{1}$, L. Muntean ${ }^{1,2}$, S. P. Simon ${ }^{1,2}$, S. Rednic ${ }^{1,2} .{ }^{1}$ County Emergency
Hospital, Rheumatology, Cluj Napoca, Romania; ${ }^{2}$ University of Medicine and Pharmacy "Iuliu Hatieganu", Rheumatology, Cluj-Napoca, Romania

Background: In the context of the coronavirus (SARS-CoV-2) pandemic, severa studies looked at the relation between rheumatic disease and SARS-CoV-2. It remains unclear whether these patients are at increased risk of developing more severe cases of coronavirus disease (COVID-19) or not.

Objectives: The objective of this descriptive study is to report the characteristics and outcomes of rheumatic patients that had a history of confirmed SARS-CoV-2 infection. Findings have also been compared to some of the existing publications on COVID-19 in these patients.

Methods: Between November 17, 2020 and January 18, 2021, a single-centre observational study was conducted in the rheumatology department of the Emergency Clinical County Hospital and the University of Medicine and Pharmacy "Iuliu Hatieganu" in Cluj-Napoca, Romania. The sample consisted of 62 rheumatic patients with a positive polymerase chain reaction test from nasopharyngeal/oral swab. Data on both systemic autoimmune disease and COVID-19 was collected using a survey, by means of telephone or in the outpatient clinic setting. Data on the patient rheumatologic condition was also collected from the electronic health records available within our department

Results: 62 patients were included, with $85.48 \%$ females and $14.51 \%$ males, and a median age of 52 years (SD +/- 14).

The most frequent comorbidities were high blood pressure (HBP) $46.77 \%$, dyslipidaemia $19.35 \%$, liver disease $17.74 \%$, and interstitial lung disease (ILD) $12.90 \%$. Recurrent COVID-19 symptoms included: cough $(62 \%)$, fever $(46,77 \%)$, anosmia $(46.77 \%)$, ageusia $(30.64 \%)$, headache $(29.03 \%)$, gastrointestinal symptoms $(27.41 \%)$ and myalgia $(25.80 \%)$.

Out of the entire 62 sample, 41 patients had an inflammatory arthritis (IA) diagnosis, with the most frequent being rheumatoid arthritis (RA) - $68.29 \%$, followed by ankylosing spondylitis $-21.95 \%$, psoriatic arthritis $-7.31 \%$ and $2.43 \%$ with Still disease. Only 10 patients suffered from connective tissue disease (CTD): 3 systemic lupus erythematosus, 2 poli/dermatomyositis, 2 Sjogren syndrome (SjS), 2 mixed connective tissue disease, 1 systemic sclerosis (SSc). Another 10 patients had overlapping syndromes with the most frequent $(40 \%)$ overlap between RA and SSc. One patient had osteoarthritis.

49 patients followed a treatment with conventional synthetic disease-modifying anti-rheumatic drugs with $51.2 \%$ of them being treated with Methotrexate.

14 of our patients received glucocorticoids (GC), but no relation between the GC dose and COVID-19 severity could be observed. Only 3 patients with doses greater than $10 \mathrm{mg} /$ day were present in the cohort and 2 developed mild while 1 developed an asymptomatic COVID-19 course.

22 patients had received biological treatment. Anti TNF alpha medication was administered to 13 of these, and mostly consisted of Adalimumab, Etanercep and Golimumab. The anti TNF alpha patients were asymptomatic or had mild forms of COVID-19 (93.30\%)

8 cases had ILD: 3 RA patients, 3 overlapping syndromes, $1 \mathrm{SSc}$ and $1 \mathrm{SjS}$. The median age was 59,5 years (SD +/- 10). $25 \%$ exhibited severe, $37,5 \%$ moderate, $25 \%$ mild and $12.5 \%$ asymptomatic COVID- 19 .

The COVID-19 severity in our sample was as follows: $12.90 \%$ of the patients were asymptomatic, $59.67 \%$ exhibited a mild form, $19.35 \%$ a moderate one, and out of the $8.06 \%$ with a severe case of COVID-19, 1 patient died. The median age in the severe cases of COVID-19 was 66 years $(S D+/-12)$ and HBP was the most common comorbidity.

Conclusion: Most patients in this sample were either asymptomatic or had a mild COVID-19 evolution. Although the research design has multiple limitations, rheumatic pathology does not seem to be a higher risk factor for severe COVID19 than other associated comorbidities. With that in mind, ILD patients should be closely monitored as even in on our limited sample size a worse evolution of COVID-19 has been observed. Biological treatments, especially anti TNF alpha might help in reducing the severity of COVID-19, but this outcome could have been associated in our sample with other factors like lower median age and less comorbidities.

Disclosure of Interests: None declared.

DOI: 10.1136/annrheumdis-2021-eular.740

\section{AB0657 \\ PERCEIVED STRESS AND FOOD INSECURITY IN PREGNANT AND POSTPARTUM WOMEN WITH RHEUMATIC DISEASES DURING THE COVID-19 PANDEMIC}

L. G. Espinosa Banuelos ${ }^{1}$, P. R. Ancer Rodríguez², M. G. Herrera López², C. M. Skinner Taylor ${ }^{1}$, L. Pérez Barbosa ${ }^{1}$, R. Moyeda Martinez ${ }^{1}$, R. A. Rodriguez Chavez $^{1}$, A. Y. Lujano Negrete ${ }^{1}$, A. Cárdenas ${ }^{1}$, D. Á. Galarza-Delgado'.

${ }^{1}$ Hospital Universitario Dr. José Eleuterio González, Servicio de Reumatología, Monterrey, Mexico; ${ }^{2}$ Hospital Universitario Dr. José Eleuterio González, Clínica de Nutrición, Departamento de Medicina Interna, Monterrey, Mexico 